\title{
Battery Aware Beacon Enabled IEEE 802.15.4: An Adaptive and Cross-Layer Approach
}

\author{
Marwa Salayma, Ahmed Al-Dubai, Imed Romdhani, Muneer Bani Yassein* \\ School of Computing, Edinburgh Napier University, Edinburgh, UK \\ \{M.Salayma; A.AL-Dubai; I.Romdhani\}@napier.ac.uk \\ *Department of Computer Science, Jordan University of Science and Technology, Irbid, Jordan \\ *masadeh@just.edu.jo
}

\begin{abstract}
In Wireless Sensor Networks $\left(\mathrm{WSN}_{\mathrm{s}}\right)$, energy conservation is one of the main concerns challenging the cutting-edge standards and protocols. Most existing studies focus on the design of WSN energy efficient algorithms and standards. The standard IEEE 802.15.4 has emerged for $\mathrm{WSN}_{\mathrm{s}}$ in which the legacy operations are based on the principle that the power-operated battery is ideal and linear. However, the diffusion principle in batteries shows the nonlinear process when it releases a charge. Hence, we can prolong the network lifetime by designing optimized algorithms that reflect the battery characteristics. Within this context, this paper proposes a cross-layer algorithm to improve the performance of beacon enabled IEEE 802.15.4 network by allowing a Personal Area Network Coordinator (PANc) to tune its MAC behavior adaptively according to both the current remaining battery capacity and the network status. The performance of the new algorithm has been examined and compared against that of the legacy IEEE 802.15.4 MAC algorithm through extensive simulation experiments. The results show that the new technique reduces significantly the energy consumption and the average end-to-end delay.
\end{abstract}

Index Terms-WSN; IEEE 802.15.4, MAC; analytical model; battery aware; delay.

\section{INTRODUCTION}

$\mathrm{R}$ ECENTLY, most wired sensors are now being replaced with wireless ones creating the emerging era of Wireless Sensor Networks (WSNs). WSNs consist of sensing devices that can communicate with each other and with the surrounding environment via the wireless communication medium [1] [2]. Yet, a huge number of sensor nodes are often scattered in unreachable areas, and WSNs are often battery powered and cannot be easily recharged. Thus, energy conservation is one of the main concerns in the area of WSN. Many studies that focus on designing WSN energy efficient algorithms and standards, based on IEEE 802.15.4, have emerged recently [3]. The IEEE 802.15.4 standard supports both physical and Media Access Control (MAC) layers. IEEE 802.15.4 MAC supports two types of devices, namely, Full Functional Devices (FFDs) and Reduced Functional Devices (RFDs). FFDs act as a regular coordinator and/or as a sink node. If both features are taken, the node is typically referred to as Personal Area Network Coordinator (PANc). In contrast, RFDs act as an ordinary end device [4]. Despite these differences, both FFDs and RFDs communicate with each other, forming two types of topologies, star and peer to peer topologies. All of the supported topologies must have one PANc. In the star topology, all network nodes can only communicate with the PANc in their active period. On the other hand, in mesh network topologies, all devices can talk to each other directly sending broadcast messages.

The IEEE 802.15.4 MAC layer operates either in beacon enabled or beaconless modes. In the beacon enabled mode, the
FFD broadcasts regular beacon frames that synchronise nodes when they need to access the channel [4]. The time between two successive beacons is referred to as the Beacon Interval $(B I)$, which is divided virtually into 16 equal sized slots. BI duration is specified by the Beacon Order parameter (BO) according to the following formula [4].

$$
\mathrm{BI}=\mathrm{aBaseSuperframeDuration} * 2^{\mathrm{BO}}
$$

Nodes can use the channel during the whole BI period or can sleep for some time portions depending on Superframe Order (SO) parameter. This parameter decides the Superframe Duration (SD) active session according to the following formula [4].

$$
\mathrm{SD}=\text { aBaseSuperframeDuration } * 2^{\text {so }}
$$

where $0 \leq \mathrm{SO} \leq \mathrm{BO} \leq 14$

The aBaseSuperframeDuration value depends on the slot duration according to the following formula.

aBaseSuperframeDuration=

aBaseslotDuration * total slots $(3)$

All these concepts can actually be indicated through one concept: the duty cycle (D). This is the percentage of time the node is awake from the whole time between the two successive beacons. D is mathematically expressed as [5][6]:

$$
\mathrm{D}=\mathrm{SD} / \mathrm{BI} * 100 \%
$$

When a node needs to access the medium, it has to locate the beginning of the next time slot in order to compete for the channel based on the Carrier Sense Multiple Access/Collision Avoidance algorithm (CSMA/CA). This time portion is referred to as the Contention Access Period (CAP) [4-6].

The lengths of the discussed periods are assigned through the beacon frame, which is transmitted in the first time slot (slot 0) [5][6]. Due to the complicated issues of the inactive period, most beacon enabled IEEE 802.15.4 studies are limited to star one-hop topology. Similarly, this paper considers the same assumption.

BO and SO values controls the performance of beacon enabled IEEE 802.15.4. Small BO values lead to frequent beacon frames and consequently increase beacon overhead, which, in return, drains more power in a short period of time. Small SO values, on the other hand, decrease nodes active time, while increasing the sleep time period. However, while small SO values might save energy, they increase delay and adversely affect throughput. This is because nodes which do not have enough time to send their data frames during the current superframe, will differ in their activity to the next superframe and therefore attempt to send their data packets in one go causing collision. Clearly, this situation becomes worse as the number of nodes increases [5][6]. Beacon overhead, collision and packets retransmission are all reasons for early battery charge depletion. In order to maximize node lifetime, we 
need to increase battery lifetime. To achieve that we need to analyse the battery behaviour and study how it cope with IEEE 802.15.4 operations. Most of the studies found in literature are based on the fact that the IEEE 802.15.4 battery operations are ideal and linear. Batteries deliver power based on the electro-chemical reactions that occur between the electrodes and the active material around the electrodes. Continuous electro-chemical reactions deplete active mass near the electrode. Active material is able to diffuse towards the electrodes when the battery is idle allowing battery to heal and gain some of its charge [7]. This is called battery recovery effect, which occurs in the idle recovery time. Accordingly, in order to provide energy, real battery behaviour is governed by complex non-linear internal chemical reactions which occur due to the nonlinear behaviour when it gains charge in the recovery time [7].

To conclude, we can prolong network lifetime through adaptive techniques designed according to the battery's behaviour. For instance, we can exploit battery recovery effect by adding a relaxation time artificially between two packets in order to gain more capacity charge. Through this way, the performance of IEEE 802.15.4 could be improved by adopting IEEE 802.15.4 battery-friendly algorithm for the packets transmission.

Nevertheless, improving battery performance should not be at the expense of the standard reliability. Packets average end to end delay can be estimated at higher layers of the protocol stack, while energy consumption and battery behaviour are evaluated at the lower layers with respect to the OSI model. Therefore, the overall IEEE 802.15.4 protocol stack needs to be revisited so that the MAC layer can adaptively tune its parameters according to the actual needs in terms of the available battery capacity and the current delay. In other words, friendly battery management technique should be able to adapt to the actual network operating status; according to average end to end delay for example. This could be achieved for example by exploiting information provided by the different layers of the protocol stack. By following a cross-layer approach we do believe that we can minimize the energy expenditure.

In this paper, we propose an adaptive and cross layer approach that improves the beacon enabled IEEE 802.15.4 performance by allowing the MAC layer to tune its parameters according to the battery behaviour of the coordinator as well as the network status in a star topology. To summarize, the contribution of this paper is fourfold:

- The real behaviour of the battery in a beacon enabled IEEE 802.15.4 MAC is investigated by considering battery nonlinearity by analysing the diffusion of chemical reactions in the battery following Rakhmatov model.

- The gain of the battery recovery effect according to what sleep period can increase battery life time of the beacon enabled IEEE 802.15.4 is analysed.

- A cross-layer and adaptive battery aware beacon enabled IEEE 802.15.4 MAC that tunes synchronization time according to current battery status is proposed.

- The network reliability is considered by checking network delay and tune nodes active period accordingly.

This paper is organized as follows. Section II summarizes some of the literature work which is closely related to the paper topic, while Section III illustrates the battery models and our followed methodology. In Section IV we evaluate and dis- cuss the performance of our proposed protocol. Section V concludes the paper and outlines future work.

\section{RELATED WORK}

Recently, there has been significant amount of studies that addressed the electro-chemical behaviour of batteries. Li et al. [8] proposed an analytical model that computes the life time of a low duty cycled star sensor network. In their model they considered nonlinearities of lithium-ion battery following Rakhmatov model [15] and they aimed to minimize the total energy consumption of the lithium-ion battery by finding the optimal idle and sleep period while guaranteeing energy efficiency, reliability and reasonable latency. In their proposal, they considered the trade-off between energy that is dissipated in sending frequent preambles and the period thereby sensors stay idle waiting for the preamble. Experimental results show that the proposed method can provide the optimal sleep or channel check intervals that maximize the lifetime of the network while guaranteeing a little latency and high reliability. However, this model target only a simplified work mechanism of MAC protocol, without giving details of battery nonlinearity effects on the proposed protocol on their network.

Li et al. [9] presented three battery aware algorithms that reduce power consumption and extend battery lifetime. Each one of the proposed schemes is targeted towards a specific application type, which are the hard real-time applications, the soft real-time applications, and the periodic applications. For hard real-time applications, Battery-friendly lazy packet algorithm is proposed to minimize battery charge consumption by allowing it drew lower current. A battery-friendly local optimization algorithm with slack time is targeted towards the soft real-time applications. For the periodic applications, a batteryaware task-scheduling algorithm is developed, which performs task rescheduling to achieve the battery friendly discharge profile. Li et al. [9] follows Rakhmatov model to depict both battery and recovery effect and nonlinearity. Simulation results demonstrated that the three battery-friendly algorithms perform better in extending lifetime of battery-operated sensor nodes as they reduce battery charge consumption.

Chau et al. [10] attempted to exploit the battery recovery effect in WSN. They empirically studied the gain at which the battery recovery effect prolongs commercial sensors lifetime. This effect has also been studied analytically corroborated by simulation. The outcome of [10] revealed that there is a saturation threshold at which the battery recovery resulted from idle listening will contribute less in improving the behaviour of the battery. Authors in [10] proposed a distributed battery aware duty cycle protocol and measure the battery runtime under both deterministic and randomized schedules. The authors in [10] studied also the trade-off between both delay and harnessing the recovery effect and suggested that we can perfectly harness battery recovery without increasing delay if we carefully adjust the sleep time period before reaching the saturation threshold. The authors in [11] derived upper bounds of battery lifetime and proposed a more energy-efficient algorithm that is aware of battery recovery effect and this is by extending the pseudo-random duty cycling scheme proposed in [11] by a forced sleep. In addition, the authors in [11] achieved analytical results that predict the average delay in sensor networks by setting the sleep duration of the RF transceiver as the saturation threshold of the battery, which can take 
the maximal advantage of the duration-dependent battery recovery effect. The authors in [11] presented also a useful tool to compromise the trade-off between increasing battery lifetime of sensor networks and the average delay of delivered packets.

Casilari et al. [12] proposed an analytical model that forecasts the minimum, mean and maximum battery lifetime of a WSN by allowing it to work under different traffic load, data rate and probability of packet loss. This is done by an experimental characterization of activity cycles battery consumption in commercial motes that follows the 802.15.4/ZigBee stack and also by measuring the current that is drained from the power source under different 802.15.4 communication operations [12]. The characterization considers the different operations required by 802.15 .4 protocol and takes into consideration the delay introduced by the CSMA/CA algorithm applied by the 802.15.4 MAC layer. The model has also been extended to cope with the extra consumption that the node re-association requires when a packet loss occurs. Mario et al. [13] proposed an adaptive and cross-layer energy-aware module for energy-efficient and reliable data collection targeted towards IEEE 802.15.4/ZigBee WSNs. The proposed module captures the packet delivery ratio at the application and configures the MAC layer parameters, which are backoff window size and the number of (re)transmissions, according to the traffic conditions in order to minimize the power consumption.

\section{BAtTery Model \& Protocol Description}

Battery is a repository of electrical charges which provides voltage and current for the components attached to it, such as radio transceiver, microprocessor, memory, sensor, etc. A battery losses charge when a load draws current from it, where the loss rate is a function of the load [7]. It is common that the Radio Frequency (RF) transceiver operations are the most energy consumable resources (even in listening mode), as compared to the processing and sensing activities [8]. As it shown in (5), the total energy $\left(E_{\text {total }}\right)$ consumed by RF energy model is the total sum of energies consumed by sensor in performing the four operations, which are: transmitting $\left(\mathrm{E}_{\mathrm{tx}},\right)$, in receiving $\left(E_{\mathrm{rx}}\right)$, being idle $\left(\mathrm{E}_{\text {idle }}\right)$ and in sleeping $\left(\mathrm{E}_{\text {sleep }}\right)$.

$$
\mathrm{E}_{\text {total }}=\mathrm{E}_{\mathrm{tx}}+\mathrm{E}_{\mathrm{rx}}+\mathrm{E}_{\text {idle }}+\mathrm{E}_{\text {sleep }} \text {. }
$$

IEEE 802.15.4 standard, as many of other previous studies, consider ideal behavior of the battery, that is, voltage stays constant over time until the moment it is completely discharged, then the voltage drops to zero, whereas the capacity is the same for all loads that the battery generates [7]. The total energy $\mathrm{E}$ in the ideal case can be calculated as follows.

$$
\mathrm{E}=\mathrm{V} \times \mathrm{C} \text {, and } \mathrm{C}=\mathrm{I} \times \mathrm{L}
$$

where $\mathrm{E}$ (Watt-hour) is the provided energy, $\mathrm{V}$ is the voltage (volt), $\mathrm{C}$ is the total capacity of the battery (Amperehour), $\mathrm{I}$ is the provided load (Ampere) and $\mathrm{L}$ is the lifetime of the battery (hour) [14].

In order to exploit battery characteristics in our protocol, we need to study its electro-chemical behaviour, which can be analysed empirically or through models [7]. Empirical analysis is time consuming and requires expensive prototyping and measurement for each alternative. Therefore, battery behaviour under various conditions of charge/discharge can be predicted through models [7-10].
Models for energy consumption and performance estimation of each system component are described in the following sub-section.

\section{A. Battery Models}

There are different models that describe the battery discharge processes. Each model type has a varying degree of accuracy and complexity [7][8]. Those models can be classified as low level electro chemical models and high level mathematical models. Electro chemical models are the least flexible and the most computation intensive, so they are sophisticated models to use for battery modeling and they are the most accurate ones. On the other hand, electrical circuit models, analytical models and the stochastic models can be easily configured for different types of batteries. Electrical circuit models are highly efficient when used for simulation but they ignore the effects of charge recovery during idle periods. The stochastic models are highly efficient for simulation and are capable of modeling rate capacity and recovery effects. Analytical models are computationally efficient, but limited in the discharge effects they model. One of these models is Sarma and Rakhmatov model which is an abstraction of a real battery [15] that we used in this work. Rakhmatov model is chosen for estimating the real residual battery capacity at a specific time, because it is the simplest accurate analytical model. Other models require solving complex Partial Differential Equations (PDEs) which are difficult to optimize [7][15]. For the model to adequately mimic real behaviour of the batteries, one can utilize this formula:

$$
\alpha=\mathrm{I}\left\lceil L+2 \sum_{1}^{\infty} \frac{1-e^{-\beta^{2} m^{2} L}}{R^{2} m^{2}}\right\rceil
$$

Where $\mathrm{I}$ is the applied load and L is battery lifetime, $\alpha$ is the capacity of the battery when it is fully charged, $\beta$ refers to battery materials diffusion around the electrolyte and measures the nonlinearity of the battery as it tells us how fast the diffusion process can keep up with the rate of discharge. The value of $\alpha$ is a battery related parameter and its value is decided by manufacture of battery designer [15]. Formula (7) indicates that the total capacity of the battery is the sum of two terms, the linear ideal behaviour plus the nonlinear behaviour. As long as $\beta$ value is large, the battery behaviour becomes closer to battery ideal effect. When $\beta$ goes to infinity, the battery works in its ideal situation. This means that the higher the value of $\beta$, the better the battery performs. The value of $\beta$ is estimated from the data sheet of the battery. For example, the data sheet of a battery might model rated capacity (in Ahr) vs. discharge current (in hour) [15]. Thus, before one can use the proposed model, the parameters need to be estimated from experimental data for the modeled battery. Simple experiments with constant loads are sufficient for estimation purposes. However, choosing the optimized values for both $\alpha$ and $\beta$ is beyond the scope of this paper.

It is important to note that the load generated from the battery is discharged according to different transceiver activities (transmission, receive and idle), each has its own time duration. Thus, the load can be depicted in the form of consecutive $N$ constant current values $I_{1}, I_{2}, I_{3}, \ldots, I_{N}$, where $I_{k}$ is the current of activity $k$ which took place at time $t_{k}$ in the duration of $\Delta \mathrm{k}=\mathrm{t}_{\mathrm{k}+1}-\mathrm{t}_{\mathrm{k}}$ [15]. Accordingly, battery capacity when it is fully charged can be depicted as follows: 
$\alpha=\sum_{k=1}^{N} I_{K} \Delta_{k}+\sum_{k=1}^{N} 2 I_{k} \sum_{m=1}^{\infty} \frac{e^{-\beta^{2} m^{2}\left(L-t_{k}-\Delta k\right)} e^{-\beta^{2} m^{2}\left(L-t_{k}\right)}}{\beta^{2} m^{2}}$

In order to calculate the remaining capacity at a specific time unit, we need first to calculate the amount of charge consumed after performing $\mathrm{M}$ activities (charge lost from the battery) which is denoted by $\sigma$ as follows:

$\sigma(t)=\sum_{k=1}^{M} I_{k} \Delta_{k}+\sum_{K=1}^{M} 2 I_{k} \sum_{m=1}^{\infty} \frac{e^{-\beta^{2} m^{2} t}\left(e^{\beta^{2} m^{2} \Delta_{k}}-1\right)}{\beta^{2} m^{2}} e^{-\beta^{2} m^{2} t_{k}}$

According to (8) and (9), the residual capacity at a specific time $t$ (the available charge) is presented here:

$$
\alpha(t)=\alpha-\sigma(t)
$$

\section{B. Battery Recovery Threshold}

Duty cycling is a technique adopted to regulate the on/off periods of the RF transceiver, while keeping the rest of sensor module on. It is important to design proper duty cycling and buffering strategies that can maximize the battery recovery effect when a transmitter moves to an inactive state during which the battery load becomes low, allowing the battery to recover. This results in extending the battery lifetime.

Before injecting this factor in order to improve the IEEE 802.15.4 performance, we need to analyse the rate at which sleep period can maximize battery lifetime. To achieve that, we followed Rakhmatov model and studied different active periods with different sleep time portions allowing the duty cycle to decrease gradually and we analysed the residual capacity in a star topology with 7 clients. Simulation parameters are depicted in Table 1 and the achieved results are depicted in Fig. 1. It can be noticed from Fig. 1 that, for all the tested active periods other than $61.44 \mathrm{~ms}$, increasing the sleep time portion by $50 \%$, and thus decreasing the duty cycle, increases the total residual capacity. On the other hand, allowing node sleep more than $50 \%$ decreases the total residual capacity. That is because the sleep time portion allows the chemical charge diffuses around the electrode which enables the battery heal and regain some of its charges because of the battery recovery effect principle. Moreover, it can be noticed that the effect of battery recovery increases as the active period increases, because for in a longer active time, node have enough time to do its activities and thus avoid other unnecessary operations such as, retransmission, which will save battery energy due to the increased residual capacity. This explains why for $61.44 \mathrm{~ms}$ active period, as the sleep time increases, total battery capacity increases for the three tested duty cycles. For this short period, a node does not have adequate time to perform its activities at all. Instead, it will keep differing its activity to the next superframe. Consequently, as all nodes will try to transmit together, this will cause frequent collision and retransmission which adversely affect network performance. It is therefore better for the node to sleep than to stay active. To conclude, in order to exploit battery recovery effect, $\mathrm{BO}$ is needed to be increased only by one as this will allow the node to operate within $50 \%$ duty cycle. $\mathrm{BO}$ can be incremented according to the current battery status. The algorithm description is presented in the following subsection.

\section{The Proposed Technique:}

Achieved results motivate us to propose a more energy-efficient duty cycling scheme by setting the sleep duration of the coordinator RF transceiver at the saturation threshold of the battery, which can take the maximal advantage of the duration-dependent battery recovery effect.

Nevertheless, improving battery performance should not be at the expense of other performance metrics. Packets end to end delay can be estimated at higher layers of the protocol stack, while energy consumption and battery behavior are evaluated at the lower layers. Therefore, the overall IEEE 802.15.4 protocol stack is needed to be considered for the MAC layer to adaptively tune its parameters according to the actual needs. In other words, friendly battery technique should be able to adapt to the actual network operating status. Through this approach, both physical and application layers cooperate with MAC layer in order to prolong network lifetime by preserving energy battery charge at the physical layer, while considering average end to end delay delay status at the application layer.

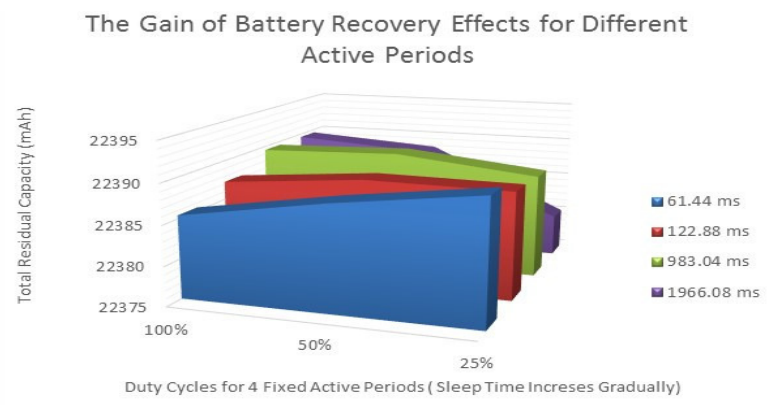

Fig. 1: Residual battery capacity for different duty cycles with different active periods.

This works as follows, before sending a new beacon frame, PANc asks the physical layer for its total residual capacity. If the new residual capacity is worse than the previous one, then it increments the value of $\mathrm{BO}$, otherwise it does nothing. At the same time, PANc also checks the number of received packets at the application layer, for example, if the number is five, and if the new average end-to-end delay is worse than the previous one, then it increments the value of SO, otherwise it does nothing. The new battery aware IEEE 802.15.4 MAC algorithm is summarized in Fig. 2.

\section{Performance evaluation}

Using QualNet 5.2 Simulator, the performance of the new proposed approach is evaluated by conducting a comparison against the legacy IEEE 802.15.4 performance in terms of total energy consumption, total battery residual capacity, average end-to-end delay and throughput. Evaluation process is applied on a star topology of seven RFDs with 7 Constant Bit Rate (CBR) traffic applications working over $1000 \mathrm{~s}$ simulation period. 
Algorithm: Battery Aware and Reliable Beacon Enabled IEEE 802.15.4 (BARBEI)

Objective: Tune MAC superframe structure parameters according to battery nonlinear behavior and network status.

Input: FFD node $f$, seven RFD nodes $r_{1}-r_{7}$

Output: New superframe structure with updated BO and SO values

Phase 1: Tune BO value according to $f$ residual capacity.

1 if $f$ send BEACON

2 if $f$. check RESIDUAL_CAPACITY $(\mathrm{t}(\mathrm{BEACON}))<$

RESIDUAL CAPACITY $(\mathrm{t}(\mathrm{BEACON}-1))=$ true

3 if BEACONN. BO! $=8$

$4 \quad \mathrm{BO}+=1$

5 endif

6 endif

Phase 2: Tune SO value according to $r_{l}-r_{7}$ average end to end delay

7 for $r_{1}$ to $r_{7}$

If $r$ check DATA PACKETS.num $\% 5=$ true

$r$. calculate (DELAY)

endif

1 endfor

12 if $f$.check AVERAGE DELAY.new > AVERAGE DE-

LAY.prevouis $=$ true

if BEACON.SO! $=$ BEACON.BO $\mathrm{SO}+=1$

endif

endif

Fig. 2: Battery Aware and Reliable Beacon Enabled IEEE 802.15.4 (BARBEI)

Table 1: QualNet 5.2 SIMULATIONPARAMETERS
\begin{tabular}{|l|l|}
\hline Parameter & Value \\
\hline Physical and MAC & IEEE 802.15 .4 \\
\hline Area & $50 \mathrm{~m} * 50 \mathrm{~m}$ \\
\hline Energy Model & MICAZ \\
\hline Number of nodes & 8 \\
\hline Transmission range & $10 \mathrm{~m}$ \\
\hline Simulation time & $1000 \mathrm{~s}$ \\
\hline Battery type & Duracell AA \\
\hline Battery model & Rhakhmatov \\
\hline Traffic & CBR $(1 \mathrm{~s}$ arrival rate $)$ \\
\hline Payload size & 50 byte \\
\hline BO values & $2,3,6,7$ \\
\hline SO Values & $2,3,4,6,7,8,9$ \\
\hline
\end{tabular}

Data rate is fixed for all nodes and the chosen packet interval is $1 \mathrm{~s}$ for a 50 bytes packet size. 16 scenarios are tested, each one with different BO: SO combination to cover different duty cycles behaviour. Each time the new algorithm performance is compared against the original IEEE 802.15.4 MAC algorithm. Each case is repeated 10 times. Simulation parameters are the same as those presented in Table 1 but with more $\mathrm{BO}$ values considered.

The following subsections illustrate the results achieved for the four metrics:
D. Total Energy Consumption $(\mathrm{mWh})$ :

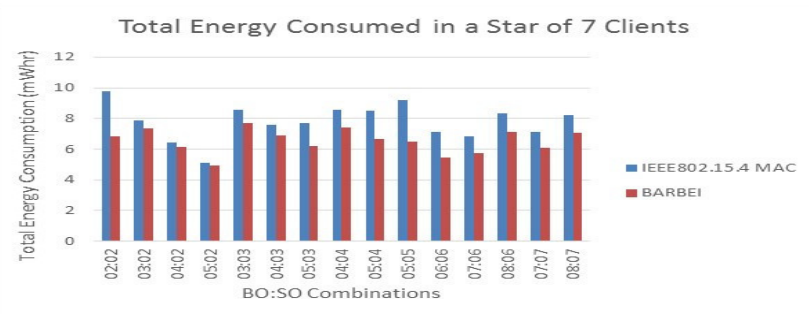

Fig. 3: Total energy consumption for a 7 RFDs in a star topology.

According to Fig. 3, it is apparent that the new algorithm decreases energy consumption regardless of the values in BO:SO combination. This is because the new algorithm tunes the MAC BO parameter according to battery residual charge. $\mathrm{BO}$ value is incremented if the current residual capacity is less than the previous one allowing the inactive period to increase. This offers node more time to sleep, which in turn allows PANc battery gain some of charge according to battery recovery effect. Consequently, battery capacity increases providing more energy according to (6). Moreover, increasing BO decreases beacon overhead which will decrease energy consumption, this effect is obviously noticed in BO: SO combination with small $\mathrm{BO}$ values, such as $\mathrm{BO}=2$. In addition, energy is saved because the new algorithm avoids packets collision and retransmissions as it increases SO according to the application layer status. This gives nodes more time to transmit packets in the increased active period.

\section{E. Residual Battery Capacity ( $m A h)$ :}

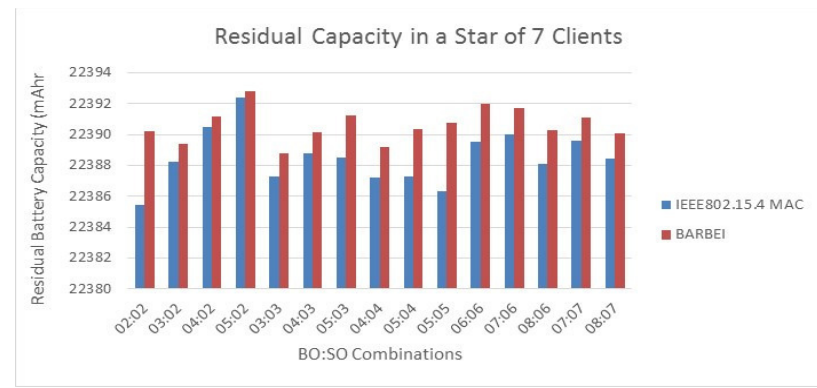

Fig. 4: Residual battery capacity for a 7 RFDs in a star topology

Fig. 4 reveals that despite the duty cycle or $\mathrm{BO}$ : SO values, the residual capacity in a network that follows our algorithm is higher than for the network that follows the legacy IEEE 802.15.4 MAC. This is because PANc exploits battery recovery effect by incrementing BO value according to battery status. This allows PANc battery to heal and gain some of its charge which will increase battery residual capacity. Network nodes are also given more time to sleep as BO increases. This saves residual battery capacity for all network nodes. Fig. 5 depicts that the new algorithm increases the throughput at most of BO:SO values. This is mostly obvious in combinations with small BO: SO values, such as 3:2, 4:2, and 5:2. In these scenarios, following the legacy MAC algorithm, the active period is two short causing a node to differ packet transmission to the next superfrme which will cause collision and hence adversely affecting network throughput. 


\section{F. Throughput (bits/s):}

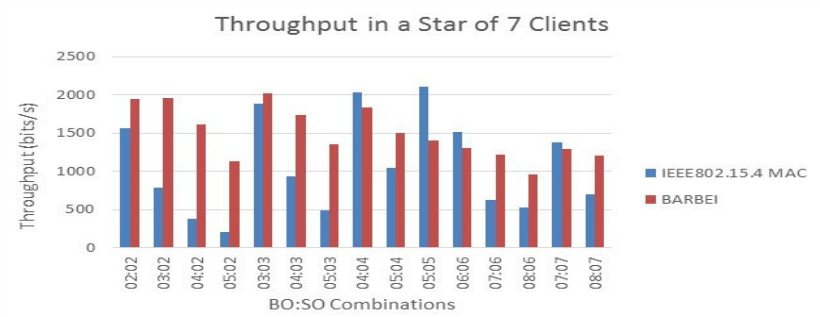

Fig. 5: Throughput for a 7 RFDs in a star topology

However, as the new algorithm allows the SO values to increase according to network performance, this will give more time for RFDs to complete their packet transmissions successfully, and consequently will improve network throughput. For combinations with $100 \%$ duty cycle, such as 4:4, 5:5 6:6 and $7: 7$, the legacy MAC outperforms our algorithm. This is because a node in these situations will have full active period to perform its activities and therefore which increase the throughput. However, increasing the inactive period according to battery status lowers the duty cycle which consequently will decrease the throughput.

\section{G. Average End To End Delay (s):}

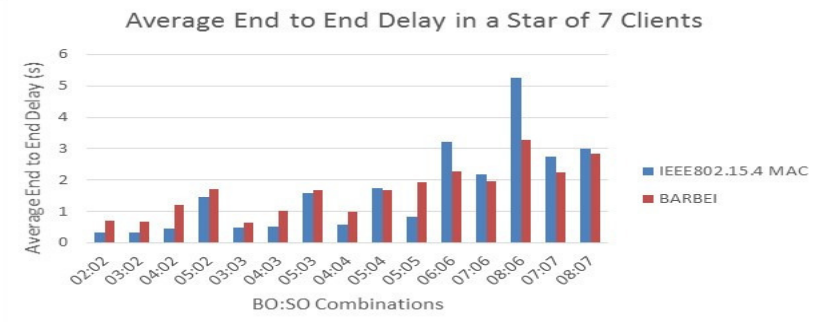

Fig. 6: Average End to End Delay for a 7 RFDs in a star topology

Fig. 6 shows that the average end-to-end delay for our algorithm performs better only for combinations with large BO values, such as $B O=6,7$ or 8 , because nodes have enough time to do their work and there is no need to increase SO value which avoids the increase of delay. Unfortunately, average end-to-end delay performs worse for the new algorithm for BO: SO combinations with small values such as $\mathrm{SO}=2,3,4$ and 5. For small BO:SO values, the delay is always bad, and there will be frequent increments in $\mathrm{BO}$ and $\mathrm{SO}$ values allowing node to operate in consequent $50 \%$ duty cycles which will increase delay.

\section{CONCLUSION}

The IEEE 802.15.4 standard is designed for different types of low-power and low-rate wireless Personal Area Networks. The performance of the standard can be improved by adopting battery-friendly algorithms for packets transmission. This can be achieved by designing battery aware approaches that exploit battery nonlinearity of recovery effects. However, there is a threshold at which battery recovery effect can be exploited. The proposed adaptive cross-layer and battery aware approach improves energy efficiency and power consumption for all possible duty cycle applications that the beacon enabled IEEE 802.15.4 standard offers. As a future work, not only the PANc is allowed to be aware of its battery behaviour, but also all network nodes will tune their activities according to their residual capacity. This can be achieved by taking the priority as criteria for packets transmission. Node priority will be determined according to its residual capacity.

\section{REFERENCES}

[1] IF. Akyildiz, W. Su, Y. Sankarasubramaniam, and A. Cayirci; “A survey on sensor networks, Communications Magazine ,"Atlanta, GA, USA, vol. 40(8), pp. 102-114, 2002. http://dx.doi.org/ 10.1109/MCOM.2002.1024422

[2] L. Selavo, A. Wood, Q. Cao, T. Sookoor, H. Liu, A. Srinivasan, and J. Porter, "wireless sensor network for environmental research", Proc. The 5 th international conference on Embedded networked sensor systems. Sydney, Australia Nov. 2007, pp. 103-116. http://dx.doi.org/10.1145/1322263.1322274

[3] A. Koubaa, "Promoting Quality of Service in Wireless Sensor Networks", Submitted for receiving Habilitation Qualification in Computer Science, National School of Engineering, Sfax, Tunisia, 2011.

[4] SC. Ergen, "ZigBee/IEEE 802.15. 4 (Summary)", [Online][accessed January 2015], Available from URL http://pages.cs.wisc.edu/ suman/courses/838/papers/zigbee.pdf.

[5] M. Salayma, W. Mardini, Y. Khamayseh, and M. Yassein, "Optimal Beacon and Superframe Orders in WSNs," in Proc. The Fifth International Conference on Future Computational Technologies and Applications (IARIA 2013), FUTURECOMPUTING 2013, Valencia, Spain, pp. 49-55, May 2013.

[6] M. Salayma, W. Mardini, Y. Khamayseh, and M. Yassein, "IEEE802. 15.4 Performance in Various WSNs Applications, "in Proc. The Seventh International Conference on Sensor Technologies and Applications, SENSORCOMM 2013, conference on Embedded networked sensor systems, Sydney, Australia, pp. 103-116, Nov. 2007. O

[7] M. R. Jongerden and B. R. Haverkort, "Battery modeling," Technical report, TR-CTIT-08-01, CTIT, 2008. http://dx.doi.org/ 10.12691/ajmo-3$2-2$

[8] Y. Li, , Y. Shouyi, 1. Leibo, W. Shaojun and W. Dong, "Battery-Aware MAC Analytical Modeling for Extending Lifetime of Low Duty-Cycled Wireless Sensor Network," in Proc. IEEE 8th Int.Conference Networking, Architecture and Storage (NAS), IEEE, pp. 297-301, 2013. http://dx.doi.org/ 10.1109/NAS.2013.47

[9] H. Li, Y. Chenfu and L. Ye, "Battery-Friendly Packet Transmission Algorithms for Wireless Sensor Networks, " Sensors Journal, IEEE 13, vol. 10, pp. 3548-3557, 2013. http://dx.doi.org/ 10.1109/JSEN.2013.2276617

[10] C. Chau, Q. Fei, S. Sayed, m. Wahab and Y. Yang, "Harnessing battery recovery effect in wireless sensor networks: Experiments and analysis, " Selected Areas in Communications, IEEE Journal on 28, vol. 7, pp. 1222-1232, 2010. http://dx.doi.org/ 10.1109/JSAC.2010.100926

[11] C. Chau, M. Wahab, F. Qin, Y. Wang and Y. Yang, "Battery recovery aware sensor networks", In Modeling and Optimization in Mobile, Ad Hoc, and Wireless Networks, 2009. WiOPT 2009. 7th International Symposium on, pp. 1-9. IEEE, 2009. Communications, IEEE Journal on 28 , vol. $7, \quad$ pp. 1222-1232, 2010. http://dx.doi.org/ 10.1109/WIOPT.2009.5291623

[12] E. Casilari, J. M. Cano-García and G. Campos-Garrido, "Modeling of current consumption in 802.15. 4/ZigBee sensor motes," Sensors, vol. 10, pp. 5443-5468, 2010. http://dx.doi.org/ 10.3390/s100605443

[13] M. Di Francesco, G. Anastasi, M. Conti, S. K. Das and V. Neri, "Reliability and Energy-Efficiency in IEEE 802.15. 4/ZigBee Sensor Networks: An Adaptive and Cross-Layer Approach, "IEEE Journal on Selected Areas in Communications, vol. 29, pp. 1508-1524, 2011. http://dx.doi.org/ 10.1109/JSAC.2011.110902

[14] D. Linden, and T. B. Reddy, "Handbook of batteries," 1985. http://dx.doi.org/10.1036/0071414754

[15] D. Rakhmatov, S. Vrudhula and D. A. Wallach, "A model for battery lifetime analysis for organizing applications on a pocket computer. Very Large Scale Integration (VLSI) Systems, "IEEE Transactions, vol. 11, pp. 1019-1030, 2003. http://dx.doi.org/10.1109/TVLSI.2003.819320 